Ann. Zootech., I972, 21 (I), 73-8I.

\title{
INFLUENCE DU SACGHAROSE SUR LE POIDS DE L'CEUF ET LA SOLIDITÉ DE LA COQUILLE. FACTEURS GÉNÉTIQUES ET NUTRITIONNELS
}

\author{
B. LECLERCQ et J.-C. BLUM \\ Station de Recherches avicoles, \\ Centre de Recherches de Tours, I. N.R. A., \\ 37 - Nouzilly \\ RÉSUMÉ
}

L'influence du saccharose sur les caractéristiques de l'œuf varie selon l'origine génétique des poules pondeuses. Dans le croisement Rhode Island $\times$ Wyandotte, les modifications sont particulièrement importantes. Les œufs sont plus gros, mais seulement au début de la ponte. La solidité de la coquille, mesurée par son index, est nettement augmentée pendant toute la durée de la ponte lorsque le régime contient $2,46 \mathrm{p}$. Ioo de calcium. Un niveau calcique élevé $(3,22 \mathrm{p}$. Ioo) augmente également la solidité de la coquille, mais supprime l'influence du saccharose. Les deux effets, sucre et calcium, ne sont donc pas additifs. Le saccharose semble agir indirectement en améliorant l'utilisation du calcium alimentaire. Actif même à petite dose (5 p. IOo), il ne peut pas être remplacé par le glucose. Dans les souches reproductrice chair naine et Leghorn, les coquilles sont moins fragiles; même pour un niveau calcique peu élevé $\left(2,4^{6} \mathrm{p}\right.$. I00), l'influence du saccharose paraît faible et inconstante.

\section{IN'TRODUC'TION}

Le sucre est bien utilisé par la Poule pondeuse (ROSENBERG, I953; Brown et al., I965). Il peut constituer jusqu'à $30 \mathrm{p}$. Ioo du régime sans que les performances de ponte soient diminuées (SMITH et KEEN, I970). Cependant, certaines caractéristiques de l'œuf peuvent être modifiées. Dans une précédente étude (LECI,ERCQ et al., I970), nous avons observé une augmentation du poids de l'œuf et de la solidité de la coquille en substituant du saccharose à l'amidon de maïs dans le régime de poules Rhode Island Red $\times$ Wyandotte. L'augmentation du poids de l'œuf ne concernait pas l'albumen mais seulement le vitellus et la coquille; elle survenait lorsque la teneur en sucre était élevée (50 p. IOo). La solidité de la coquille mesurée par son index $\left(\mathrm{g} / \mathrm{dm}^{2}\right)$ était nettement améliorée, que le régime contienne 25 ou $50 \mathrm{p}$. Ioo de sucre. 
Ces résultats peuvent être interprétés en supposant que l'action du sucre sur le métabolisme est analogue chez la Poule et chez les Mammifères : augmentation de la lipogenèse (CoLTAR'T et CROSSLEY, I970) expliquant la formation de gros jaunes ; meilleure absorption du calcium (VAUGHAN et FILER, I960) et donc solidité de la coquille accrue. Nous tentons maintenant de préciser l'effet du sucre chez la Poule pondeuse. Disposant de 3 souches différentes, nous faisons varier le taux de calcium et la dose de saccharose incorporée dans le régime. Nous comparons également les effets du glucose et du saccharose introduits à la place de l'amidon.

\section{MATÉRIEL E'T MÉTHODES}

$$
\text { r. --. Animaux et régimes }
$$

On dispose de 40 poules dans chacun des trois croisements commerciaux suivants : croisement Rhode Island Red $\times$ Wyandotte $\left(\mathrm{M}_{4} \mathrm{I}\right.$ ) destiné à la production d'œufs de consommation à coquille colorée; croisement à base de White Rock nanifié par le gène de nanisme (JV I5) et utilisé pour la production de poussins de chair ; croisement de Leghorn commercialisé pour la pro duction d'œuf de consommation à coquille blanche. Nées à la même date, les poulettes issues des croisements sont placées dans des cages individuelles à l'âge de 20 semaines. Toutes sont nourries et abreuvées à volonté ; elles sont éclairées I 6 heures par jour. Du fait de la mortalité et des arrêts de ponte, les I 20 poules ne sont pas utilisées dans chaque essai.

TABLEAU I

Composition des régimes (cn p. Ioo)

\begin{tabular}{|c|c|c|c|c|c|c|}
\hline & $\mathrm{S}_{\mathbf{0}}$ & $\mathrm{S}_{0} \mathrm{Ca}$ & $\mathrm{S}_{25}$ & $\mathrm{~S}_{25} \mathrm{Ca}$ & $S_{5}$ & $\mathrm{G}_{25}$ \\
\hline \multirow{2}{*}{\multicolumn{7}{|c|}{$\begin{array}{l}\text { Mélange commun de pro- } \\
\text { téines, vitamines et }\end{array}$}} \\
\hline & $7: 3$ & 73 & 7.3 & 73 & 73 & 73 \\
\hline Amidon de maïs & 25 & 25 & 0 & 0 & $\because 0$ & 0 \\
\hline Saccharose. & 0 & 0 & -15 & 25 & 5 & 0 \\
\hline Cellulose & 2 & 0 & 2 & 0 & 2 & 2 \\
\hline Carbonate de calcium & 0 & 2 & 0 & 2 & 0 & 0 \\
\hline Glucose (Cérélose) $\ldots \ldots$. & 0 & 0 & 0 & 0 & 0 & 25 \\
\hline Teneur en calcium $(\%)$ & 2,16 & 3,22 & 2,46 & 3,29 & 2,46 & 2,46 \\
\hline
\end{tabular}

(1) Composition du mélange commun (par $100 \mathrm{~kg}$ ): Farine de soja: $16,5 \mathrm{~kg}$ farine de poisson : $2,75 \mathrm{~kg}$; gluten de maïs : $15,1 \mathrm{~kg}$; huile de maïs : $1,35 \mathrm{~kg}$; farine de luzerne : $10,9 \mathrm{~kg}$; phosphate bicalcique : $2,75 \mathrm{~kg}$; chlorure de sodium $0,25 \mathrm{~kg}$; bicarbonate de sodium : $0,10 \mathrm{~kg}$; carbonate de calcium : $6,15 \mathrm{~kg}$; amidon de maïs : $\left\{2,5 \mathrm{~kg}\right.$; vitamine $\mathrm{A}: 1100000 \mathrm{UT}$; vitamine $\mathrm{D}_{3}$ : $200000 \mathrm{UI}$; riboflavine : $1 \mathrm{~g}$; vitamine $\mathrm{E}: 4 \mathrm{~g}$; chlorure de choline : $75 \mathrm{~g}$; DL-méthionine : $70 \mathrm{~g}$; carbonate de magnésium : $16 \mathrm{~g}$; oxyde de magnésium : $20 \mathrm{~g}$; sulfate de fer : $15 \mathrm{~g}$; sulfate de zinc : $30 \mathrm{~g}$; sulfate de manganèse : $40 \mathrm{~g}$; sulfate de cuivre $: 0,5 \mathrm{~g}$; avoine broyée : $745 \mathrm{~g}$. 
Chaque expérience comporte deux périodes. La période préexpérimentale dure 28 jours. Toutes les poules reçoivent alors le régime témoin $\left(\mathrm{S}_{0}\right)$ qui contient comme glucides l'amidon de maïs et une quantité de calcium à peine suffisante $(2,46$ p. Ioo). Au cours de lá période expérimentale, également de 28 jours, les animaux clu lot témoin continuent de recevoir le même aliment $S_{0}$ tandis que, dans les autres lots, les modifications du régime sont limitées, autant que faire se peut, aux seuls facteurs nutritionnels étudiés.

Pour chaque période, les i 8 premiers jours sont considérés comme une phase d'adaptation. Les diverses mesures sont effectuées au cours des io derniers jours. Pour chaque poule, on compare les données de la période expérimentale à celles de la période préexpérimentale. Les trois essais se succédant, les poules sont âgées de 30 semaines au début du premier, 40 semaines au début du second et 50 semaines lorsque commence le troisième.

Expérience $n^{\circ} 1$. - Cette expérience a pour but de déterminer l'effet du saccharose en fonc tion de la teneur du régime en calcium et du type de pondeuse. Les régimes expérimentaux sont $\mathrm{S}_{0} \mathrm{Ca}, \mathrm{S}_{25}$ et $\mathrm{S}_{25} \mathrm{Ca}$ (tabl. I). $\mathrm{S}_{0} \mathrm{Ca}$ ne diffère de $\mathrm{S}_{0}$ que par substitution de 2 p. Ioo de carbonate de calcium à 2 p. Ioo de cellulose de façon à élever le taux calcique de 2,46 à 3,22 p. Ioo. $\mathrm{S}_{25}$ et $\mathrm{S}_{25} \mathrm{Ca}$ proviennent respectivement de $\mathrm{S}_{\mathbf{0}}$ et $\mathrm{S}_{0} \mathrm{Ca}$ en remplaçant l'amidon de maïs par du saccharose.

Cet essai ne concerne que deux croisements commerciaux, Rhode Island Red $\times$ Wyandotte et White Rock nanifiéc, à raison de 32 sujets par croisement, soit 8 poules de chacun d'entre cux par lot.

Expérience $n^{\circ} 2$. - Grâce à cette expérience, on compte préciser l'effet de la đose đe saccharose incorporé au régime. Dans les deux régimes expérimentaux $S_{5}$ et $S_{25}$, on remplace respectivement 5 et 25 p. Ioo d'amidon par une proportion égale de saccharose. Chaque lot comprend 8 poules de chacun des trois croisements.

Expérience $n^{\circ} 3$. - Deux sucres différents, le saccharosc ct le glucose (cérélose) sont comparés dans cet essai au taux d'incorporation de 25 p. Ioo. Vingt-sept poules de chaque croiscment, soit 9 animaux par lot, sont mises en expérience.

Tous les aliments ont la même teneur en énergie métabolisable. Ils renferment une même fraction commune apportant minéraux, vitamines et protéines (tabl. I). Le saccharose utilisé est du saccharose pur non dénaturé.

\section{2. - Méthodes analytiques}

Les ceufs sont pesés individuellement le lendemain du jour de ponte. Leurs composants sont séparés, le jaune et la coquille sont pesés, le premier après avoir été débarrassé des chalazes, la seconde après lavage et séchage à l'étuve à $105^{\circ} \mathrm{C}$ pendant 24 heures. Le poids de l'albumen est obtenu par différence. L'index de coquille (poids en grammes par décimètre carré de surface) est estimé par la méthode de Mongin (I965).

L'analyse de variance est réalisée sur les différences entre les mesures effectuées sur les derniers cufs de la période expérimentale et la moyenne des 4 derniers oufs de la période préexpérimentale.

\section{RÉSULTATS}

L'effet des traitements est apprécié en comparant les variations qui apparaissent dans chaque lot expérimental d'une part et dans le lot témoin $\left(S_{0}\right)$ d'autre part.

\section{Essai no 1: Influence du saccharose et du niveau calcique de la ration}

Le tableau 2 indique les variations d'index de coquille et du poids moyen de l'œuf selon les régimes et les croisements commerciaux utilisés. Ces résultats sont enregistrés au début de la période de ponte ( $3^{\mathrm{e}}$ mois). Dans le cas des Leghorn, le niveau de la production d'œufs étant insuffisant, nous ne faisons pas état des valeurs obtenues. 


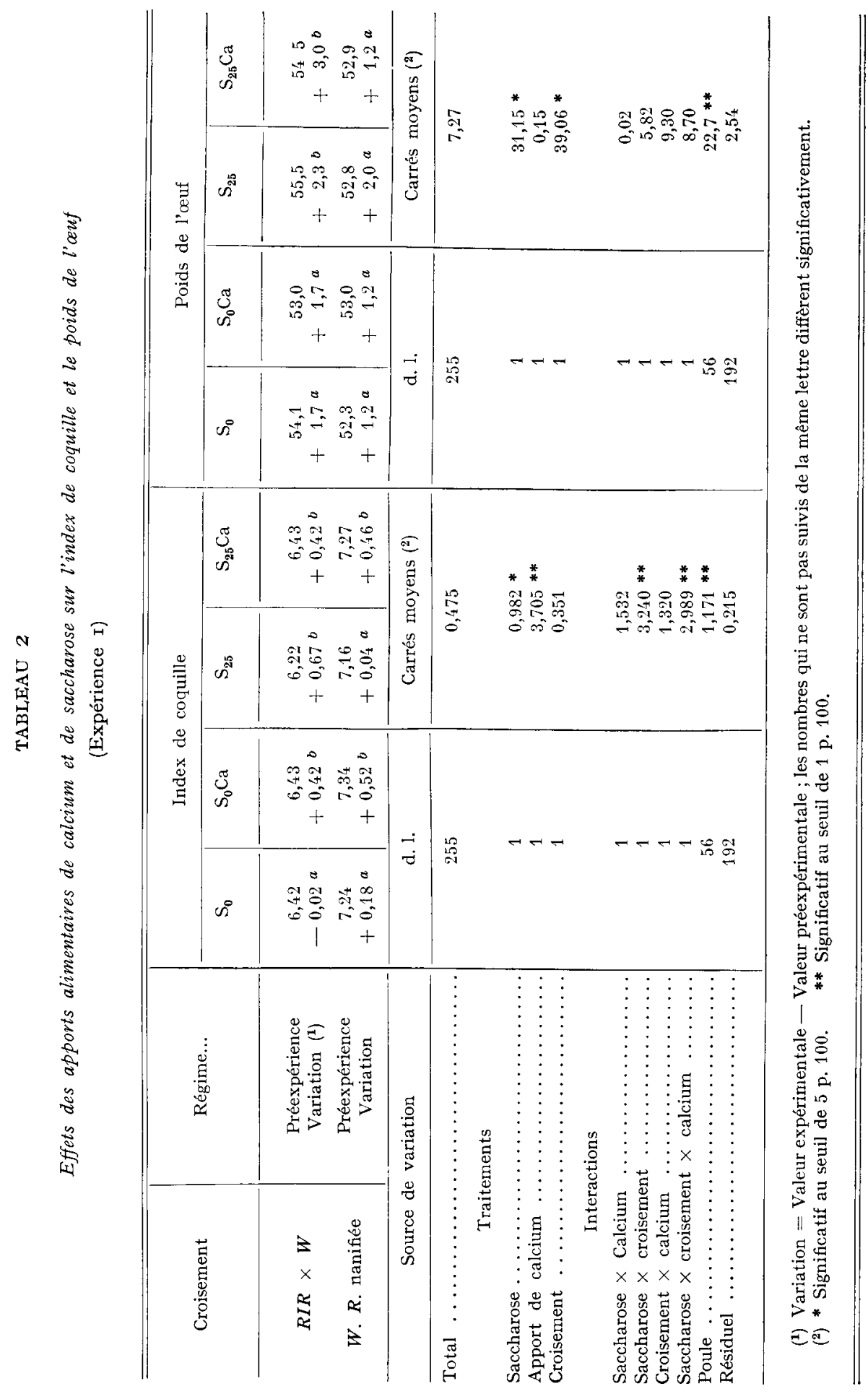


TABI,EAU 3

Effet des apports alimentaires de calcium et de saccharose sur la consommation quotidienne (Expérience I)

(g/poule/j)

\begin{tabular}{|c|c|c|c|c|c|}
\hline Croisement & Régime... & $\mathrm{S}_{0}$ & $\mathrm{~S}_{0} \mathrm{Ca}$ & $\mathrm{S}_{25}$ & $\mathrm{~S}_{25} \mathrm{Ca}$ \\
\hline $\begin{array}{c}R I R \times W \\
W . R . \text { nanifiée }\end{array}$ & $\begin{array}{c}\text { Préexpérience } \\
\text { Variation }\left({ }^{1}\right) \\
\text { Préexpérience } \\
\text { Variation }\end{array}$ & $\begin{array}{l}122 \\
-5^{a} \\
118^{a} \\
+9^{a}\end{array}$ & $\begin{array}{r}146 \\
-13 a \\
105 \\
+\quad 9 a\end{array}$ & $\begin{array}{c}140 \\
+\quad 5 b \\
109 \\
+\quad 14 a\end{array}$ & $\begin{array}{l}124 \\
+8 b \\
107 \\
+5 a\end{array}$ \\
\hline
\end{tabular}

( $\left.{ }^{1}\right)$ Variation $=$ Valeur expérimentale - Valeur préexpérimentale.

Les nombres qui ne sont pas suivis de la même lettre sont significativement différents $(\mathrm{P}<0,01)$.

\section{TABLEAU 4}

Effets de la teneur du régime en saccharose sur l'index de coquille et le poids de l'ouf

(Expérience 2)

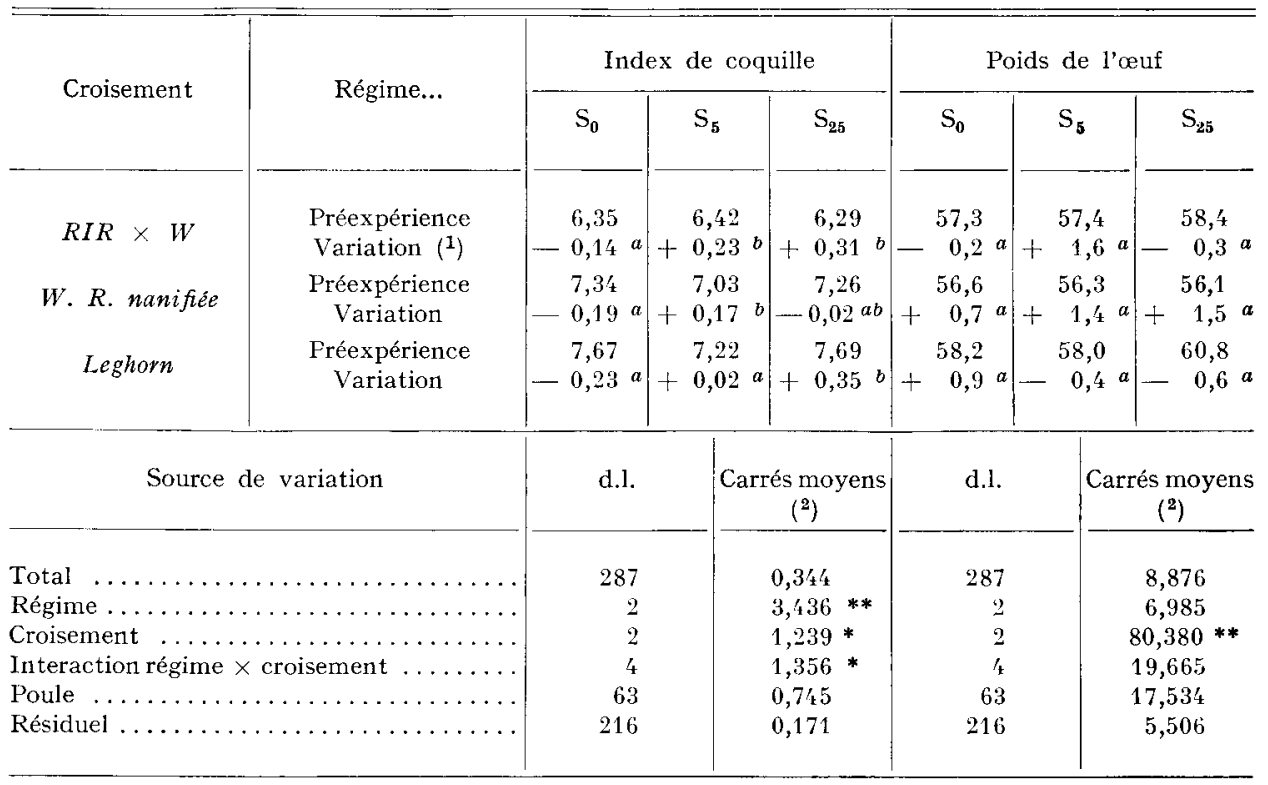

(1) Variation $\Longrightarrow$ Valeur expérimentale - Valeur préexpérimentale.

Les nombres qui ne sont pas suivis de la même lettre diffèrent significativement $(P<0,01)$

(2) * Significatif au seuil de 5 p. $100 \quad$ ** Significatif au seuil de 1 p. 100. 
On constate, en ce début de ponte, que le saccharose augmente le poids moyen de 1'œuf, particulièrement chez les poules Rhode Island Red $\times$ Wyandotte, le calcium n'ayant aucun effet.

Chez les Rhode Island Red $\times$ Wyandotte, l'index de coquille est également augmenté par un apport alimentaire de saccharose ou par un accroissement du niveau calcique de la ration $(3,22 \mathrm{p}$. Ioo contre $2,46 \mathrm{p}$. Ioo). Mais ces deux effets ne sont pas additifs et on enregistre une variation identique dans les 3 lots $\mathrm{S}_{25}$, $\mathrm{S}_{0} \mathrm{Ca}$ et $\mathrm{S}_{25} \mathrm{Ca}$. Chez les reproductrice chair JV I5, l'index de coquille dépend seulement de la teneur en calcium du régime; le saccharose n'a pas d'influence. Cet ensemble de résultats se traduit par une interaction de $2^{\mathrm{e}}$ ordre significative sucre $x$ calcium $\times$ souche.

Le tableau 3 montre que la consommation alimentaire est accrue par le saccharose chez les seules poules Rhode Island Red $\times$ Wyandotte.

\section{Essai no 2: Influence de la teneur du régime en saccharose}

L'essai no 2 est réalisé au cours du $5^{\mathrm{e}}$ mois de ponte. A cette époque, le poids de l'œuf paraît indépendant de la nature du régime (tabl. 4). Par contre, l'index de coquille augmente chez toutes les poules ingérant du saccharose. Dans le croisement

TABLEAU 5

Comparaison entre les effets du saccharose et du glucose de l'aliment sur l'index de coquille et le poids de l'auf

(Expérience 3)

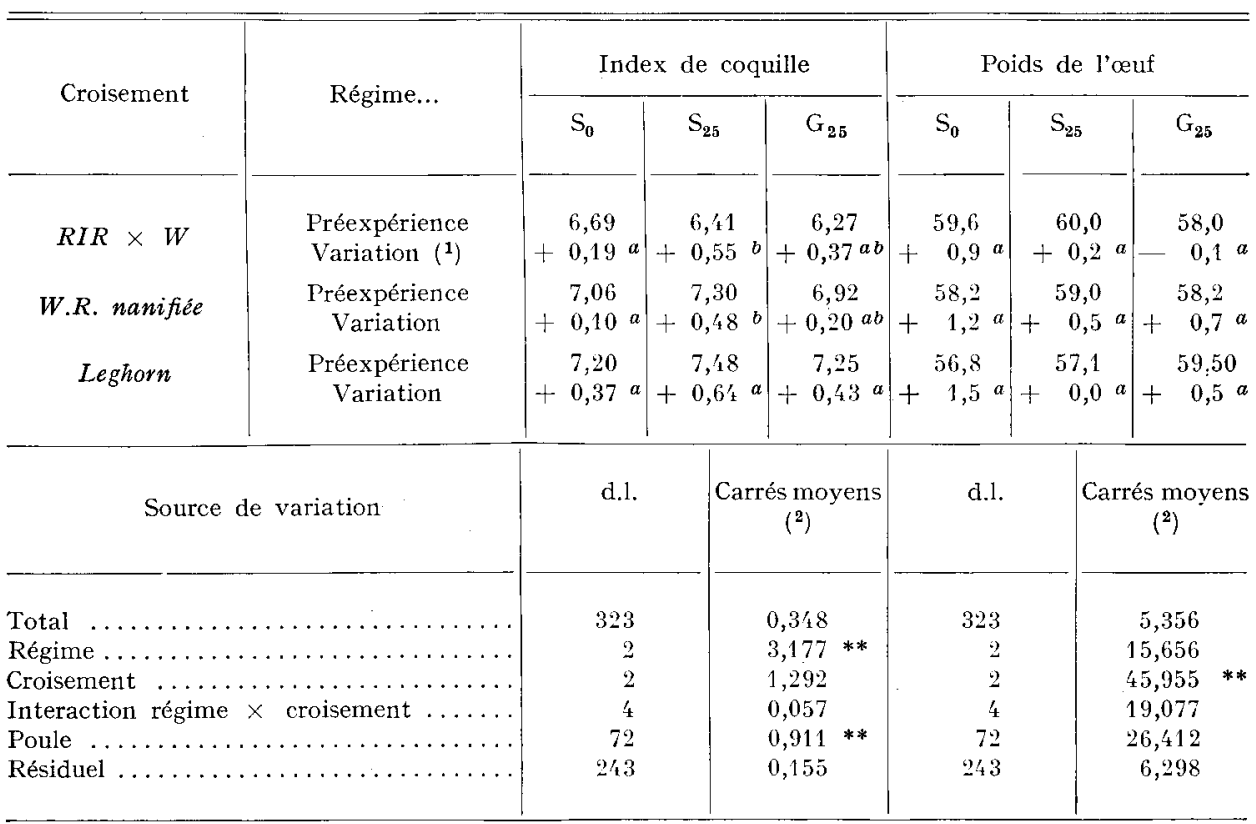

( $\left.{ }^{1}\right)$ Variation $=$ Valeur expérimentale - Valeur préexpérimentale Les nombres qui ne sont pas suivis de la même lettre diffèrent significativement

(2) Significatif au seuil de 1 p. 100 
Rhode Island Red $\times$ Wyandotte, la différence avec les témoins $\left(\mathrm{S}_{0}\right)$ est toujours significative quelle que soit la concentration du saccharose. Chez les reproductrice chair naine JV I5, l'influence du sucre est paradoxalement plus marquée pour une concentration faible: significative avec $5 \mathrm{p}$. Ioo de saccharose, non significative avec $25 \mathrm{p}$. Ioo. Chez les Leghorn, le seuil de signification $(\mathrm{P}<0$,oI) est atteint pour une teneur de 25 p. 100.

Les consommations journalières d'aliments ne sont pas indiquées pour cet essai car elles ne varient pas significativement sous l'effet du saccharose.

\section{Essai no 3 : Influence comparée du saccharose et du glucose}

Lorsque les poules ingèrent du saccharose, $l$ 'index de coquille augmente significativement dans les trois croisements commerciaux (tabl. 5). Cependant, l'effet est moins significatif chez les Leghorn $(\mathrm{P}<0,05)$ que dans les deux autres croisements $(\mathrm{P}<0,01)$. L'influence du glucose sur l'index paraît semblable mais moins marquée que celle du saccharose; elle n'est pas significative.

Enfin, il faut remarquer que les deux sucres (saccharose et glucose) ne modifient ni le poids moyen de l'œuf (tabl. 5) ni la consommation alimentaire.

Les résultats de ces 3 essais confirment dans une large mesure nos premières observations (L,ECLERCQ et al., I970) ; ils permettent, en outre, de préciser dans quelles conditions le saccharose modifie les caractéristiques de l'œuf.

Dans le cas du poids de l'œuf nous avions observé une augmentation en substituant le sucre à l'amidon après plusieurs mois de ponte chez les poules Rhode Island $\times$ Wyandotte. Peut-être parce que le régime est différent (moins de sucre et moins de protéines), cette influence du saccharose apparait seulement lors du premier essai, au début de la ponte. L'action du saccharose sur le poids de l'œuf dépend donc de facteurs génétiques et nutritionnels, mais aussi de la période de ponte.

\section{DISCUSSION}

L'index mesurant la solidité de la coquille (HunTon, I969; Mongrn, I969), sa variation présente un intérêt zootechnique certain et mérite tout particulièrement d'être commentée. Dans le croisement Rhode Island Red $\times$ Wyandotte il est également amélioré par une augmentation de la teneur du régime en calcium ou par la substitution du sucre à l'amidon. Les deux effets ne sont pas additifs. L'intervention du saccharose ne résulte pas d'un changement de la consommation alimentaire : une augmentation est observée dans le I er essai, mais, identique dans les 2 lots $S_{25}$ et $S_{25} \mathrm{Ca}$, elle parait surtout liée à l'augmentation du poids de l'œuf. Du reste, chez les poules témoins, il n'existe aucune corrélation entre la variation de consommation et la variation d'index de coquille.

Malgré les nombreux travaux effectués chez les mammifères, aucune explication satisfaisante n'a pu être apportée à l'influence des différents glucides sur 1'utilisation digestive des minéraux (FÉVRIER et RÉRAT, I964; FourNIER et DUPUIS, I968; Pansu et Chapuy, I970 ; LABat, 1971). Dans notre essai, le saccharose semble supprimer un facteur limitant de l'utilisation du calcium lorsque la teneur de l'aliment est basse ; ce facteur limitant n'existe plus lorsque le niveau calcique est élevé $(3,2$ p. Ioo 
chez les Rhode Island $\times$ Wyandotte). Analysant l'effet du lactose sur 1'absorption intestinale du calcium chez le Rat, FINL,AYSON (I970) suppose que le calcium peut être absorbé sous deux ou plusieurs formes qui diffèrent par leur activité métabolique. Chez la Poule, une de ces formes, sensible à l'action du saccharose, interviendrait particulièrement dans la formation de la coquille. Cette forme de transport serait saturée lorsque le niveau calcique est élevé. Dans le croisement Rhode Island $\times$ Wyandotte, il faut 3,2 p. Ioo de calcium pour que la saturation soit atteinte, le saccharose est actif à faible dose ( 5 p. Ioo), il ne peut pas être remplacé par le glucose. Chez les poules reproductrices naines et chez les Leghorn, le saccharose a une influence plus faible : ou bien la forme de transport est moins sensible à l'action du sucre, ou bien elle est déjà presque saturée lorsque le niveau calcique atteint $2,46 \mathrm{p}$. Ioo.

Sur le plan pratique, l'intérêt du saccharose est variable selon la souche. L'accroissement du poids de l'œuf peut être recherché, surtout au début de la ponte. Mais, l'action la plus constante du saccharose concerne la solidité de la coquille. Une augmentation du niveau calcique pourrait provoquer une baisse de performance et une mauvaise absorption des oligo-éléments (SCHAIBLE et BANDEMER, I94I ; TURK et al., I959) et de ce fait, il serait préférable d'introduire du sucre dans l'aliment lorsque l'index de coquille est bas.

Reçu pour publication en juillet 1971.

\section{SUMMARY}

EFFEC' OF SUCROSE ON THE EGG WEIGHT AND EGG-SHELI, STRENGTH.

GENETIC AND NUTRITIONAL, FACTORS

The influence of sucrose on the characteristics of the egg varied according to the genetic origin of the laying hens. The changes observed in Rhode Island Red $\times$ Wyandotte crosses were particularly interesting. The eggs were heavier, but only at the beginning of the laying period. The egg-shell strength, measured by the shell index, was much higher during the whole laying period when the diet contained $2.46 \mathrm{p}$. I oo calcium. A high calcium level (3.22 p. I00) also increased the egg shell strength, but the influence of sucrose was suppressed. Consequently, the two effects, sugar and calcium, were not additive.

Sucrose seems to act indirectly, improving the efficiency of the dietary calcium. Being active event at a low level ( 5 p. Ioo), it cannot be replaced by glucose. As regards the breeding strains dwarf White Rock and Leghorn, the egg shells were less fragile; even at a rather low calcium level $(2.46 \mathrm{p}$. Ioo), the influence of sucrose appeared to be small and inconstant.

\section{RÉFÉRENCES BIBLIOGRAPHIQUES}

Brown W. O., Waring J. J., Souance E., I965. A study of the effect of variation in the calorieprotein ratio of a medium energy diet and a high energy diet containing sucrose on the efficiency of egg production in caged layers. Brit. Poult. Sci, 6, 59-66.

Coltart T. M., Crossley J. N., I97o. Influence of dietary sucrose on glucose and fructose tolerance and triglyceride synthesis in the baboon. Clin. Sci., 33, 427-437.

Février C., Rérat A., I964. Influence du lactose sur la croissance et la composition corporelle du Rat blanc. I. Relation avec le taux protidique de la ration. Ann. Biol. anim. Bioch. Biophys., 4, 423-439.

Finlayson B., I97o. Lactose and intestinal absorption of calcium. Invest. Urology, 7, 433-441.

Fournier P., Dupuis Y., I968. Les tissus calcifiés. S. E. D. E. S. (Paris), 283 p.

Hull S. J., Scott M. L., I 969 . A further study on the calcium requirements of laying hens. Proc. Cornell Nutr. Conf., 6z-64. 
Hunton P., I969. The measurement of egg shell strength : a comparison of four methods. Brit. Poult. Sci., 10, $28 \mathrm{I}-289$.

LABAT M. L., I97r. Effet de l'actinomycine D sur l'absorption intestinale de calcium provoqué par le lactose, C. R. Acad. Sci., 272, 2005-2008.

Leclerco B., Mongin P., Blum J. C., r97o. Effets d'une substitution réciproque entre le saccharose et l'amidon sur le poids du jaune et du blanc de l'ouf et sur la qualité de la coquille. Ann. Zootech., 19. I9-25.

Mongrin P., I965. Index de solidité de la coquille. Ses significations. Sa précision. Ann. Zootech., 14, 319-325.

Mongin P., I969. Comparaison entre 3 méthodes de mesture de la solidité de la coquille. Ann. Zootech., 18, 359-36r.

Pansu D., Chapuy M. C., x97o. Relation chez l'homme entre l'action sur la calcémie de divers composés glucidiques et leur utilisation digestive. C. R. Acad. Sci., 270, 3103-3106.

Rosenberg M. M., I953. Low grade sugar, a potential carbohydrate feedstuff for laying chickens. Poult Sci., 32, 69-77.

Shaible P. J., Bandemer S. L., I94I. The effect of mineral supplements on the availability of manganese. Poult. Sci., 21, 8-I4.

SMith A. J., KEEN P., I97o. The use of sugar as a replacement for maize in diets for laying pullets. World's Poult. Sci. J., 26, 7o6.

Turk D. E., Sunde M. L., Hoknstra W. G., I959. Zinc deficiency experiments with poultry. Poult. Sci, 38, 1256.

Vaughan O. W., Filer L. J., 1960. Enhancing action of certain carbohydrates on the intestinal absorption of calcium in the Rat. J. Nutr., 71 , I0-14. 